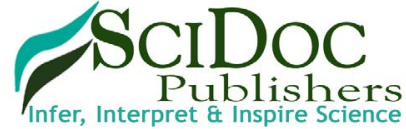

International Journal of Anesthesiology \& Research (IJAR)

ISSN 2332-2780

\title{
A Randomized Parallel Controlled Study of the Efficacy and Safety of Lornoxicam Versus Etoricoxib after Total Knee Arthroplasty
}

Research Article

Cionac Florescu $\mathrm{S}^{1 *}$, Anastase $\mathrm{DM}^{1}$, Munteanu $\mathrm{AM}^{1}$, Porumbac $\mathrm{G}^{1}$, Mihailide $\mathrm{N}^{2}$

${ }^{1}$ Department of Anaesthesiology, Clinical Hospital of Orthopaedics Foisor, Bd. Ferdinand nr. Bucharest, Romania.

${ }^{2}$ Department of Orthopaedics, Clinical Hospital of Orthopaedics Foisor, Bd. Ferdinand nr. Bucharest, Romania.

\section{Abstract}

Objectives: The aim of the study was to compare the postoperative analgesic efficacy and safety of lornoxicam versus etoricoxib for the first 48 hours after surgery.

Methods: We conducted a prospective randomized controlled study on 110 patients ASAI-II scheduled for TKA under spinal anesthesia, who received either lornoxicam $8 \mathrm{mg}$ PO at the end of surgery and a further $8 \mathrm{mg}$ after 12 hours (Lornoxicam Group) or etoricoxib $120 \mathrm{mg}$ at the end of surgery and one placebo pill after 12 hours (Etoricoxib Group). The primary outcome measure was the cumulative dose of morphine administered during the first postoperative 24 and 48 hours. Secondary outcomes were duration of analgesia and the side effects of the treatment.

Results: The groups were similar in terms of demographic data. There are no significant differences between groups regarding the morphine consumption at 24 hours (36.2 \pm 12 in Lornoxicam group and $34.5 \pm 14.1$ in Etoricoxib group) and 48 hours postoperatively (15.6 \pm 12.8 in Lornoxicam group and $18 \pm 12.3$ in Etoricoxib group) or between the duration of analgesia (314.5 \pm 70.4 in Lornoxicam group and $320.4 \pm 89.2$ in Etoricoxib group).

Conclusion: Postoperative use of lornoxicam for 48 hours in the dose of $8 \mathrm{mg}$ PO twice a day in patients undergoing TKA has an analgetic effect comparable to etoricoxib $120 \mathrm{mg}$, fewer patients experienced adverse symptoms in the etoricoxib group, but the difference was not statistically significant.

Keywords: TKA; Postoperative Pain; Lornoxicam; Etoricoxib.

\section{Introduction}

Pain relief after total knee arthroplasty continues to be a subject of debate. Although there are a variety of pharmacological therapies, no ideal drug has been found to date $[1,2]$.

Non-steroidal anti-inflammatory drugs (NSAIDs) are commonly used for the treatment of post-operative pain in order to reduce opioids administration and their adverse effects $[3,4]$.

Cyclooxygenase-2 (COX-2) specific inhibitors are advantageous over the previous generations of NSAIDs in terms of safety, since they have significantly less gastrotoxicity and no effects on platelet aggregation, important in the postoperative setting $[5,6]$.

Etoricoxib [7] belonging to the second class of selective inhibitors of COX-2, has a good absorption rate and a half-life of 22 hours that allows administration of a single $120 \mathrm{mg}$ tablet daily. Literature data show that its absorbtion is complete in about 24 minutes after administration [8] and its postoperative use as an analgetic was demonstrated to be efficient and safe. Rawal et al., used postoperative etoricoxib 90 and $120 \mathrm{mg}$ in patients undergoing TKA and found it superior to placebo and non-inferior to ibuprofen in reducing pain at rest and morphine consumption [9].

Rasmussen et al., used etoricoxib $120 \mathrm{mg}$ daily on 228 patients with knee and hip arthroplasty and showed it provided analgesia similar to naproxen sodium $1100 \mathrm{mg}$ at the first 24 hours after surgery and superior to placebo, with reduced opioid medication [10].

Lornoxicam [7] is a potent new NSAIDs oxicam derivative, which

\footnotetext{
*Corresponding Author:

Simona Florescu Cionac,

Department of Anaesthesiology, Clinical Hospital of Orthopaedics Foisor, Bd. Ferdinand nr. 35-37, 021382, Bucharest, Romania.

Tel: 0040722381861

E-mail: florescut@yahoo.com

Received: November 21, 2016

Accepted: December 15, 2016

Published: December 17, 2016

Citation: Cionac Florescu S, Anastase DM, Munteanu AM, Porumbac G, Mihailide N (2016) A Randomized Parallel Controlled Study of the Efficacy and Safety of Lornoxicam Versus Etoricoxib after Total Knee Arthroplasty. Int J Anesth Res. 4(12), 373-376. doi: http://dx.doi.org/10.19070/2332-2780-1600077

Copyright: Cionac Florescu S ${ }^{\circ}$ 2016. This is an open-access article distributed under the terms of the Creative Commons Attribution License, which permits unrestricted use, distri bution and reproduction in any medium, provided the original author and source are credited.
} 
exerts its analgesic effect via inhibition of cyclooxygenase I and II and also by release of endogenous dynorphins and beta-endorphins.

It is available for parenteral and enteral administration. It has a relatively rapid on set of action compared to other oxicams and a shorter half-life (approximately 4 hours) that explains its improved gastrointestinal safety profile $[11,12]$.

The aim of this study was to demonstrate the analgesic efficacy and safety profile of lornoxicam administered postoperatively after TKA performed under spinal anaesthesia versus etoricoxib.

\section{Material and Method}

Ethical approval of the study was provided by the Ethics Committee of the Orthopedics Hospital Foisor, Bucharest, Romania (Protocol ID AN-002-13).

After obtaining the written informed consent, a total number of 110 patients were assigned to the study, having the inclusion criteria: ASA score I-II, age 30-80 years, Body Mass Index (BMI) $20-30$, Hct $\geq 30 \%$ and indication of primary TKA.

Patients were not eligible for the study if they had history of bleeding disorders, peptic ulcer or gastrointestinal bleeding, severe liver and kidney dysfunction, bronchial asthma, congestive heart failure (NYHA II-IV), neuropathies, allergies to the drugs used in the study, psychiatric disorders, chronic treatment with corticosteroids or long-acting NSAIDs administered in the last 4 days preoperatively, as well as those with cerebrovascular or peripheral arterial disease or arterial hypertension not adequately controlled.

Patients who met the criteria were enrolled and randomized by a computer generated random number list (http:/ /www.graphpad. com/quickcalcs), conducted by an investigator who was not involved in this study. The result placed inside a numbered envelope (one number corresponding to one patient of the study). Another investigator also not involved in treating patients opened the sealed envelope and administered the pills, according to patient's allocation group:

- LORNOXICAM group with 55 patients who received one pill of $8 \mathrm{mg}$ PO at the end of surgery, immediately after arrival in the postoperative care unit (PACU) and a second pill after 12 hours.

- ETORICOX group with 55 patients who received a pill of 120 $\mathrm{mg}$ PO at the end of surgery, also immediately after arrival in PACU and a second placebo pill after 12 hours.

Before initiation of the neuraxial block, patients received into the pre-anesthesia area a sodium chloride $0.9 \%$ infusion at a rate of $250 \mathrm{ml} \mathrm{h}^{-1}$ containing the antibiotic for surgical prophylaxis on a 16-gauge peripheral vein catheter (Vasofix ${ }^{\circledR}$ BBraun, Melsungen, Germany). All patients were premedicated with $0.03 \mathrm{mg} / \mathrm{kg} \mathrm{IV}$ Midazolam (Dormicum ${ }^{\circledR}$, Roche, Switzerland). Spinal anesthesia was performed with $0.5 \%$ isobaric plain bupivacaine $0.3 \mathrm{mg} / \mathrm{kg}$ using 25 or 27 pencil-point gauge Whitacre needles, the spinal puncture taking place in the sitting position at the L3-L4 intervertebral space.
At 15-20 minutes after the injection of the local anesthetic, motor block was assessed by Bromage scale and sensory block level was tested by loss of sensation to pinprick. Intraoperative sedation was provided with boluses of IV $10-20 \mathrm{mg}$ of propofol to maintain a 3 sedation level on a Ramsay scale [13]. Electrocardiogram in D2 and V5 leads, peripheral oxygen saturation $\left(\mathrm{SpO}_{2}\right)$ and non-invasive blood pressure automatically every 5 minutes were monitored by Draeger (Draeger Medical Systems Monitor, Draeger AG, Luebeck, Germany) during the surgery ; the urinary output was recorded orally. Also the body temperature (axillar) was maintained at $36.5^{\circ} \mathrm{C}$ by an electric warming blanket (BairHugger $\left.^{\circledR}\right)$. Oxygen was administered at $5-6 \mathrm{~L} \mathrm{~min}^{-1}$ by a facemask.

At the end of the surgery the patients were taken to the PACU for the next $48 \mathrm{~h}$ where they were monitored as intraoperative. The pills were administrated according to randomization. During the postoperative period, the patients were asked to quantify their pain using the numerical rating scale (NRS, from 0 - no pain - to 10 -the most pain possible).

The multimodal analgesia plan was started when NRS $>3$ with the administration of paracetamol (Perfalgan ${ }^{\circledR}$, Bristol- Myers Squibb, New York, USA) $1 \mathrm{~g}$ IV in 15 minutes every 8 hours for the next 48 hours followed by administration of morphine (Morphine hydrochloride ${ }^{\circledR}$, Sanofi Aventis Zentiva, Paris, France) if NRS persists to remain above 3 , with a loading bolus of $0.1 \mathrm{mg} /$ $\mathrm{kg}$ IV supplemented with $2 \mathrm{mg}$ IV every 5 minutes until NRS < 3 according to the IV titration protocol approved and applied in PACU of our hospital. This analgesia protocol was applied until 48 hours from end of surgery. Ondansetron $4 \mathrm{mg}$ IV was administered before the first dose of morphine to all patients (Osetron $^{\circledR}$, Dr. Reddy 's, India).

Adverse effects such as respiratory depression, nausea, vomiting, sedation, bradycardia, dizziness, urinary retention after removal of the catheter, pruritus or desaturation were recorded throughout the study. Sedation was evaluated by Ramsay scale. Respiratory depression was considered at a breathing rate $\leq 8 / \mathrm{min}$ and treated with naloxone. In case of $\mathrm{SPO}_{2} \leq 95 \%$, oxygen was given on a facemask. Heart rate $<50 /$ min was considered as bradycardia and treated with IV atropine $10 \mu \mathrm{g} / \mathrm{kg}$. Hypotension was defined as MAP $<70 \mathrm{mmHg}$ and treated with IV ephedrine 5-10 $\mathrm{mg}$ IV and saline solution.

The following outcome parameters were monitored during the study:

a. Primary outcome: The cumulative morphine $(\mathrm{mg})$ consumed during the first 24 hours and 48 hours postoperatively to maintain NRS $\leq 3$, at rest;

\section{b. Secondary outcomes:}

b.1. The duration of analgesia defined as the time from the initiation of spinal anesthesia until the first analgesic requirement (min);

b.2. The side effects of the treatment for 48 hours.

All parameters were evaluated by medical staff not aware of the patients randomization.

The statistical analysis was performed using GraphPad Prism 6.0 
(GraphPad Software, Inc, La Jolla CA 92037, USA).

For an alpha type (false positive) error rate of $0.05 \%$ and a beta type (false negative) error rate of $90 \%$, assuming a mean value of morphine consumption of $40 \mathrm{mg} \pm 20 \mathrm{mg}$ (SD) in the etoricoxib group (14.15), and a reduction with $30 \%$ of this value in the lornoxicam group, the sample size needed was 58 patients in each of the two groups. (http://clincalc.com/Stats/SampleSize).

Continuous variables (age, BMI, quantity of morphine used, duration of analgesia) are presented as means \pm SD when distribution was found normal, or median $\pm 25-75 \%$ quartiles when distribution was nonsymmetric. The two groups were compared using Mann-Whitney test. Nominal data (gender, side effects) are presented as frequency tables and compared using Fisher's exact test. A value of $\mathrm{p}<0.05$ was considered statistically significant.

\section{Results}

In the period september 2014 - april 2015 out of the 124 patients meeting the inclusion criteria, a number of 14 were excluded because of requesting another type of anesthesia or refusal to sign informed consent. The groups were similar in terms of ASA class, age, BMI, gender, number of patients, surgery type and duration, data being presented as mean \pm standard deviationin Table 1 .

At 24 hours after surgery no statistically significant difference was noted between groups regarding the morphine consumption, 36.2 $\pm 12 \mathrm{mg}$ in Lornoxicam group, and respectively $34.5 \pm 14.1 \mathrm{mg}$ in Etoricoxib group; also, at 48 hours postoperatively the morphine administered was $15.6 \pm 12.8 \mathrm{mg}$ in Lornoxicam group and $18 \pm$ $12.3 \mathrm{mg}$ in Etoricoxib group, with no significant difference be- tween groups ( $p=0.265$ and 0.326 respectively).

The duration of analgesia was not different between the 2 groups: $314.5 \pm 70.4 \mathrm{~min}$ in Lornoxicam group and $320.4 \pm 89.2 \mathrm{~min}$ in Etoricoxib group $(\mathrm{P}=0.708)$, the results being presented as mean \pm standard deviation in Table 2 .

The overall incidence of adverse events in the 2 groups during the first 48 hours is shown in Table 3 . The tolerability of etoricoxib appeared to be superior to lornoxicam, with less number of patients experiencing adverse drug reactions.

\section{Discussion}

The results of the present study showed no significant difference regarding the duration of analgesia and morphine consumption at 24 and 48 hours postoperatively after TKA when lornoxicam or etoricoxib were administered at the end of surgery, immediately after the patients arrived in the PACU. Lomoxicam was well tolerated in this study and although fewer patients experienced adverse effects in Etoricoxib group, the differences are not statistically significant.

Clinical trials that evaluate the effectiveness and safety of lornoxicam as part of a multimodal analgesic regimen in reducing postoperative pain and decreasing the need for rescue medications in orthopaedic and other types of surgery are becoming more numerous [14-16].

The general precautions with regard to the use of NSAIDs and the potential risks, like gastrointestinal bleeding, kidney problems and/or cardiovascular side effects must be acknowledged.

Table 1. Demographic Data (Mean \pm SD).

\begin{tabular}{|c|c|c|}
\hline & Etoricoxib Group & Lornoxicam Group \\
\hline Number & 55 & 55 \\
\hline Age (year) & $66.12 \pm 8.5$ & $64.2 \pm 7.3$ \\
\hline BMI & $29 \pm 2$ & $29 \pm 7$ \\
\hline Sex (F/M) & $44 / 11$ & $45 / 10$ \\
\hline Duration of surgery & $123 \pm 20$ & $122 \pm 22$ \\
\hline
\end{tabular}

Table 2. Morphine Requirements (Mean \pm SD) and Time Lasting Analgesia (Mean \pm SD).

\begin{tabular}{|c|c|c|c|}
\hline Morphine Requirements (mg) & Etoricoxib Group & Lornoxicam Group & $\boldsymbol{P}_{\text {value }}$ \\
\hline Surgery day & $34.5 \pm 14.1$ & $36.2 \pm 12$ & NS \\
\hline Day one & $18 \pm 12.3$ & $15.6 \pm 12.8$ & NS \\
\hline Lasting analgesia (min) & $320.4 \pm 89.2$ & $314.5 \pm 70.4$ & NS \\
\hline
\end{tabular}

Table 3. The Number and Percentage of Patients with Side Effects.

\begin{tabular}{|c|c|c|c|}
\hline & Etoricoxib Group & Lornoxicam Group & $P$ value \\
\hline Nausea & $2 / 55(1.1 \%)$ & $4 / 55(2.2 \%)$ & NS \\
\hline Vomiting & $1 / 55(0.5 \%)$ & $3 / 55(1.6 \%)$ & NS \\
\hline Sedation & $1 / 55(0.5 \%)$ & $1 / 55(0.5 \%)$ & NS \\
\hline Bradycardia & $2 / 55(1.1 \%)$ & $2 / 55(1.1 \%)$ & NS \\
\hline Dizziness & $0 / 55$ & $0 / 55$ & NS \\
\hline Urinary retention & $0 / 55$ & $0 / 55$ & NS \\
\hline Pruritus & $0 / 55$ & $1 / 55(0.5 \%)$ & NS \\
\hline Desaturation & $0 / 55$ & $0 / 55$ & NS \\
\hline
\end{tabular}


In a randomized, placebo controlled study conducted by Inan et al., lornoxicam in a dose of $24 \mathrm{mg}$ in the surgery day and $8 \mathrm{mg}$ for the next 24 hours reduced morphine consumption and side effects related to morphine without additional side effects of lornoxicam after total knee replacement [17].

Sivrikoz et al., reported an improved analgesia and a decreased morphine consumption following hip and knee arthroplasty after IV postoperative administration of lornoxicam $8 \mathrm{mg}$ twice a day or dexketoprofen $50 \mathrm{mg}$ twice a day [18].

The introduction of selective inhibitors of COX-2 has provided new options in the postoperative pain management of patients because coxibs are not associated with most of the side effects of NSAIDs like platelet dysfunction and stomach problems.In a study published in 2013 Lin et col. have shown the advantages of perioperative administration of COX-2 inhibitors [19].

Studies comparing non-selective with selective NSAIDs to treat acute postoperative pain are rare [20]. White et al., conducted a randomized controlled study to evaluatethe effects of postoperative administration of a nonselective nonsteroidal anti-inflammatory drug, ibuprofen, in a dose of $1200 \mathrm{mg}$ /day versus celecoxib, a cyclooxygenase- 2 selective inhibitor,in a dose of $400 \mathrm{mg} /$ day on the postoperative pain and the need for analgesics medication in ambulatory surgery. Both anti-inflammatory drugs significantly decreased the need for opioids in the early postdischarge period, leading to an improvement in the quality of recovery and patient satisfaction [21].

In another prospective, randomized, double-blinded, placebocontrolled study Papadima compared the efficacy of lornoxicam versus parecoxib for the management of pain after laparoscopic cholecystectomy on 76 patients and concluded that lornoxicam $8 \mathrm{mg}$ i.v. provided statistically equivalent pain relief to that of parecoxib $40 \mathrm{mg}$ i.v. and both were more efficacious than placebo [22].

Our prospective double-blinded randomized study investigated the postoperative analgetic efficacy of lornoxicam versus etoricoxib. To our knowledge this is the first study comparing lornoxicam and etoricoxib in postoperative pain management.

We preferred to study etoricoxib because this drug has a rapid bioavailability via the oral route and the half-life longer than other coxibs could be an advantage by extending the analgetic effect. On the other hand, lornoxicam has a rapid onset of action compared to other oxicams, which it is also an important factor in the immediate postoperative period.

The present study has certain limitations because all parameters evaluated in the study were followed up for 48 hours only and no long-term data about pain, analgesic requirements or the safety of the drug given perioperatively were given. Also, we evaluated the pain only at rest; thus, it remains uncertain whether the results would be similar during the rehabilitation methods and recovery.

\section{Conclusion}

Postoperative use of lornoxicam for 48 hours in the dose of $8 \mathrm{mg}$
PO twice a day is safe, effective and comparable to etoricoxib 120 $\mathrm{mg}$ in postoperative pain relief after TKA.

\section{References}

[1]. Rawal N (2016) Current issues in postoperative pain management. Eur J Anaesthesiol. 33(3): 160-71.

[2]. Elmallah RK, Cherian JJ, Pierce TP, Jauregui JJ, Harwin SF, et al., (2016) New and Common Perioperative Pain Management Techniques in Total Knee Arthroplasty. J Knee Surg. 29(2): 169-78.

[3]. Joshi GP, Viscusi ER, Gan TJ, Minkowitz H, Cippolle M, et al., (2004) Effective treatment of laparoscopic cholecystectomy pain with intravenous followed by oral COX-2 specific inhibitor. AnesthAnalg. 98(2): 336-342.

[4]. Ng A, Temple A, Smith G, Emembolu J (2004) Early analgesic effects of parecoxib versus ketorolac following laparoscopic sterilization: a randomized controlled trial. Br J Anaesth. 92(6): 846-849.

[5]. Goldstein JL, Correa P, Zhao WW, Burr AM, Geis GS, et al., (2001) Reduced incidence of gastroduodenal ulcers with celecoxib, a novel cyclooxygenase- 2 inhibitor, compared to naproxen in patients with arthritis. Am J Gastroenterol. 96(4): 1019-1027.

[6]. Harris SI, Stoltz RR, LeComte D, Hubbard RC (2004) Parecoxib sodium demonstrates gastrointestinal safety comparable to placebo in healthy subjects. J Clin Gastroenterol. 38(7): 575-580.

[7]. Arcoxia 30mg, $60 \mathrm{mg}, 90 \mathrm{mg} \& 120 \mathrm{mg}$ Film-coated Tablets (2013) Summary of Product Characteristics last updated on the eMC: 12/08.

[8]. Takemoto JK, Reynolds JK, Remsberg CM, Davies NM, Vega-Villa (2008) Clinical pharmacokinetic and pharmacodynamic profile of etoricoxib. Clin Pharmacokinet. 47(11): 703-720.

[9]. Rawal N, Viscusi E, Peloso PM, Minkowitz HS, Chen L, et al., (2013) Evaluation of etoricoxib in patients undergoing total knee replacement surgery in a double-blind, randomized controlled trial. BMC Musculoskelet Disord. 24(14): 300 .

[10]. Rasmussen GL, Malmstrom K, Bourne MH, Jove M, Kotey P, et al., (2005) Etoricoxib provides analgesic efficacy to patients after knee or hip replacement surgery: a randomised double-blind, placebo-controlled study. Anesth Analg. 101(4): 1104-1111.

[11]. Hitzenberger G, Radhofer-Welte S, Takacs SF, Rosenow D (1990) Pharmacokinetics of lornoxicam in man. Postgrad Med J. 66(4): 22-6.

[12]. Radhofer-Welte S, Rabasseda X (2000) Lornoxicam, a new potent NSAID with an improved tolerability profile. Drugs Today. 36(1): 55-76.

[13]. Ramsay MA, Savege TM, Simpson BR, Goodwin R (1974) Controlled sedation with alphaxalone-alphadolone. Br Med J. 2(5920): 656-659.

[14]. Hillstrom C, Jakobsson JG (2013) Lornoxicam : pharmacology and usefulness to treat acute postoperative and musculoskeletal pain a narrative review. Expert Opin Pharmacother. 14(12): 1679-94.

[15]. Karaman Y, Kebapci E, Gurkan A (2008) The preemptive analgesic effect of lornoxicam in patients undergoing major abdominal surgery: a randomised controlled study. Int J Surg. 6(3): 193-196.

[16]. KorkmazDilmen, Ozlem; Tunali, Yusuf; Cakmakkaya, Ozlem S (2010) Efficacy of intravenous paracetamol, metamizol and lornoxicam on postoperative pain and morphine consumption after lumbar disc surgery. Eur J Anaesthesiol. 27(5): 428-432.

[17]. Inan N, Ozcan N, Takmaz SA, Ozcan A, Erdoğan I, et al., (2007) Efficacy of lornoxicam in postoperative analgesia after total knee replacement surgery. Agri. 19(2): 38-45.

[18]. Sivrikoz N, Koltka K, Güresti E, Büget M, Sentürk M, et al., (2014) Perioperative dexketoprofen or lornoxicam administration for pain management after major orthopedic surgery: a randomized, controlled study. Agri. 26(1): 23-8.

[19]. Lin J, Zhang L, Yang H (2013) Perioperative administration of selective cyclooxygenase- 2 inhibitors for postoperative pain management in patients after total knee arthroplasty. J Arthroplasty. 28(2): 207-213.

[20]. Wickerts L, Warrén Stomberg M, Brattwall M, Jakobsson J (2011) Coxibs: is there a benefit when compared to traditional non-selective NSAIDs in postoperative pain management? Minerva Anestesiol. 77(11): 1084-98.

[21]. White PF, Tang J, Wender RH, Zhao M, Time M, et al., (2011) The effects of oral ibuprofen and celecoxib in preventing pain, improving recovery outcomes and patient satisfaction after ambulatory surgery. Anesth Analg. 112(2): 323-329.

[22]. Papadima A, Lagoudianakis E, Antonakis PT, Pattas M, Manouras A, et al., (2007) Parecoxib vs. lornoxicam in the treatment of postoperative pain after laparoscopic cholecystectomy: a prospective randomized placebo controlled trial. Eur J Anaesthesiol. 24(2): 154-158. 